\title{
A Prototype of a Robot Capable of Tracking Anyone with a High Body Temperature in Crowded Areas
}

https://doi.org/10.3991/ijoe.v17i11.25463

\author{
Mohamed Zied Chaari $\left.{ }^{\bowtie}\right)$, Abdulaziz Aljaberi \\ Qatar Scientific Club, Doha, Qatar \\ chaari_zieddieee.org
}

\begin{abstract}
People's health and the world economy around worldwide are under the impact of the virus. Controlling the propagation of coronavirus is extraordinarily challenging after the seventeen months since the pandemic outbreak. This research will represent the efficiency of robots in helping humanity in facing the Corona pandemic and the global health crisis. According to the quick and widespread Covid-19 contagion globally, healthcare service demand increased staff with less availability. In this crisis, robotics is the safe solution at a low price to support people to fight Covid-19. Furthermore, it is essential to relax lockdown restrictions after the increase in the number of people vaccinated. In this context, we designed a mobile robot with a thermal temperature scanner for pedestrians and identified those with abnormal temperatures over $39^{\circ} \mathrm{C}$ in public places and track them in real-time. This robot features a long-lasting, lightweight battery and a high-quality thermal camera. Our primary target is to reduce the risk of transmission between people. We use a single-board computer and a thermal camera to detect unusual fevers near real-time to achieve this design and prototyping. In addition, we use image processing to detect target pedestrians and a control system to guide the robot. This robot can bypass any potential obstacles that can prevent it from moving around and cause problems. The development of this robot mainly focuses on the real-time measurement of body temperature at two meters. The compensation algorithm can coordinate between the mechanical parts of the robot and high-efficiency design.
\end{abstract}

Keywords-human tracking, image processing, covid-19, thermal scanner, robotic, $3 \mathrm{~d}$ printing

\section{$1 \quad$ Introduction}

The Covid-19 virus has changed our daily life and habits. Most people want to go back to their pre-Covid lives and look back at their regular living days once the lockdown is lifted [1], [2]. However, things are not expected to be as they in a short time [3], [4]. By 2021, rich countries began vaccinating their citizens at a very high rate. Reports issued by the World Health Organization (WHO) say that the organization will continue to apply preventive measures such as masks and social distancing in effect in every area of life despite the increase in the number of people who have been vaccinated in recent months [5]-[7]. It was clear that the pandemic caused significant losses in the 
leading economies of rich and developed countries separately. Now, a year and a half into the pandemic, we can begin to compare the performance of international economies and the policy response [8]-[10]. The United Nations (UN) estimates that the pandemic will quickly push an additional 100 million people into poverty. Many research and laboratories worldwide work together to find solutions to mitigate the pandemic's significant social and economic impact and help people travel and return to their preCovid lives. In most countries, vaccine supplies are stratified by age, prioritizing the elderly at the most significant risk of dying from Covid-19 [11], [12]. Several countries, such as Israel, began vaccinating their citizens at the end of 2020, and thanks to a deal with Pfizer-BioNTech to share statistics for vaccine doses, it currently leads the world in the number of people vaccinated [13], as shown in Figure 1. About 50\% of the country's population fully vaccinated with the two doses required for protection as of midMarch. The Israeli government started to relax lockdown restrictions a month later as vaccinations against the Covid-19 virus were rolled out more widely [14].

\section{Countries with a population of at least one million}

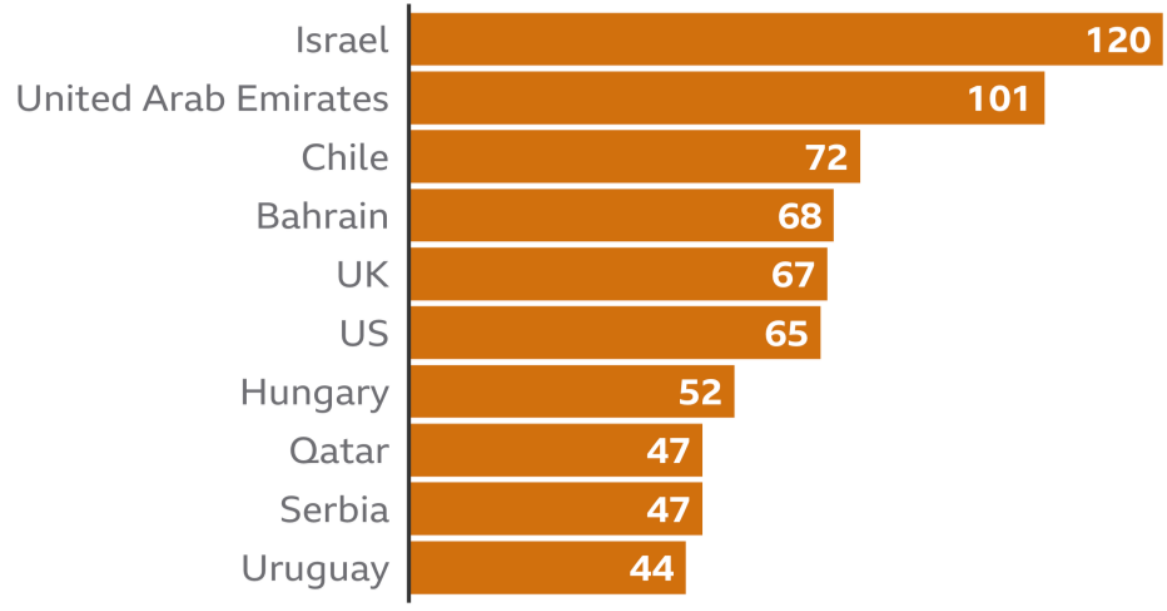

Note: Total vaccinations refers to the number of doses given, not necessarily the number of people vaccinated

Source: Our World In Data, 12:00 BST on 23 Apr

Fig. 1. Total vaccine doses per 100 people

Meanwhile, neighboring countries such as Lebanon, Syria, Jordan, and Egypt have not vaccinated even $1 \%$ of their population. Qatar and the UAE have also vaccinated many of their residents and have already begun easing lockdown restrictions. Where the Qatari Ministry of Public Health (MOPH) has confirmed, the total number of vaccine doses that were administered amounted to about 3,938,599 in August 2021 [15], [16], as shown in Figure 2; despite the boost in the number of vaccinated populations, the Ministry maintained the same procedures, including masks, hand hygiene, and social distancing. 

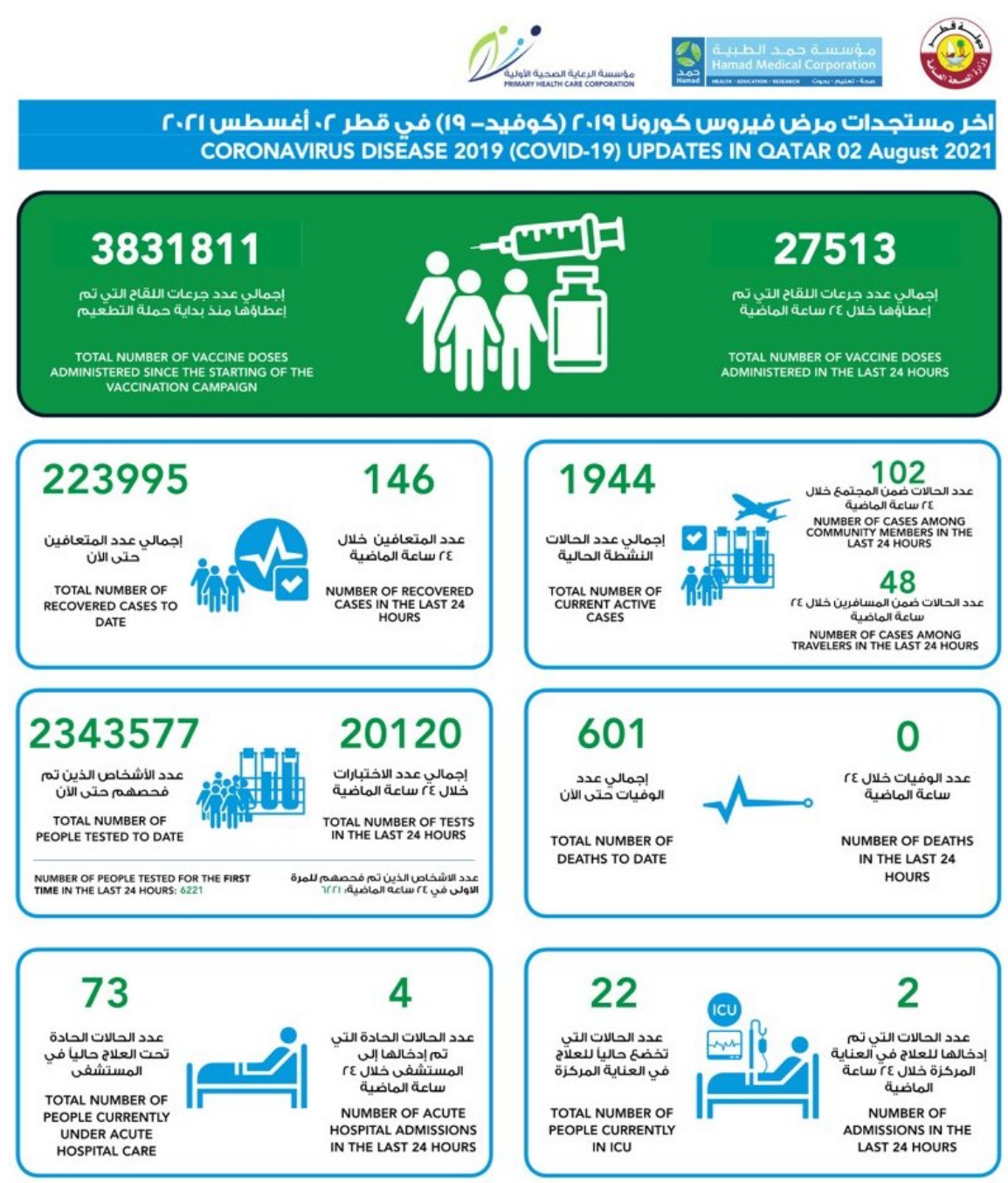

TOTAL NUMBER OF INFECTED PEOPLE SINCE THE START OF THE EPIDEMIC : 226540 For more info. on Coronsuirus Disease 2019 (ConD-19) visit www.moph.gov.qa or call 16000

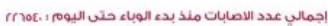

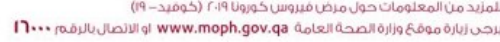

Fig. 2. Total vaccine doses per 100 people [17], [18]

Therefore, the number of people getting vaccinated is increasing worldwide, so many governments begin to open public areas and relax lockdown restrictions. However, the World WHO authorities want people to wear masks and social distancing, even after receiving a vaccine. This means that the vaccine is not enough, and governments must prepare and use advanced technology in this context. In 2020, a team at Qatar Science Club (QSC) succeeded in manufacturing a Powered air-purifying respirator (PAPR) [19]. Recently, the Fab-Lab laboratory developed a robot to identify and track individuals with fever in commercial complexes, government buildings, or any 
public area [20]. Visual manipulation of images captured around the surroundings is crucial. Therefore, this robot designed mainly for two proposals:

First, a constant temperature measurement model and we create an intelligent algorithm to determine pedestrians' temperature inside the building. Second, when identifying a person with a high temperature, the robot forces them to immediately go to the health care center to check the cause of the abnormal body temperature. Finally, since the start of the Covid-19 pandemic, we can see thermal cameras deployed in several locations to determine the temperature of people suspected of having a spotted fever. It is installed at various entry points such as airports, metro stations, auditoriums, government buildings, and hospitals to determine body temperature from two meters after lifting the closure. In addition, thermal camera screening uses for preliminary detection of the virus.

Our project primarily uses the MLX90640-D55, with a resolution of $24 \times 32$, which gives each frame 768 different temperature readings and primarily uses it as an image processing program to track and target abnormal temperatures [21]-[23]. The MLX90640 IR thermal camera panels contain a 24x32 array of infrared thermal sensors. When connecting the camera to a single board computer, they will return a set of 768 different infrared temperature interpretations via the $\mathrm{I} 2 \mathrm{C}$ communication interface [24]. In addition, the thermal camera is an embedded panel for simple and easy integration into a pandemic prevention robot (CPR).

The MLX90640 IR thermal camera's temperatures ranged from $-40 \mathrm{~F}$ to $572 \mathrm{~F}$ with a resolution of $\pm 29 \mathrm{~F}$ [25]. The maximum frame rate of MLX90640 equals $16 \mathrm{~Hz}$, which is ideal for abnormal fever temperature [26]. Programmers can even perform high-precision processing with the help of SciPy python library and good results on a single board [27], [28]. This camera reads the information in a checkerboard pattern twice per frame, so it is normal to see the gradient effect of the checkerboard when the camera moves in a specific direction. However, this effect is not remarkable when objects stir slowly. The primary purpose of our project was to design and manufacture a robot that could detect and track pedestrians with fever and unusual fever while in motion, as shown in Figure 3.

The thermal camera and computer unit work in unison to detect and track pedestrians with high body temperatures. Therefore, this robot can track moving pedestrians. The prototype contains several parts: the mechanical platform, the electronic circuit, the temperature rises detection, and the intelligent tracking system - the schematic diagram of the many parts that make up this prototype, as shown in Figure 4.

This manuscript is structured as follows: Section 1 is the introduction. Then section 2 , we study the human body temperature. After section 3, we present the system design and implementation. Then in section 4 , we discuss the robot prototype and results. Finally, we finalize a prototype device discussion and conclude the future work. 


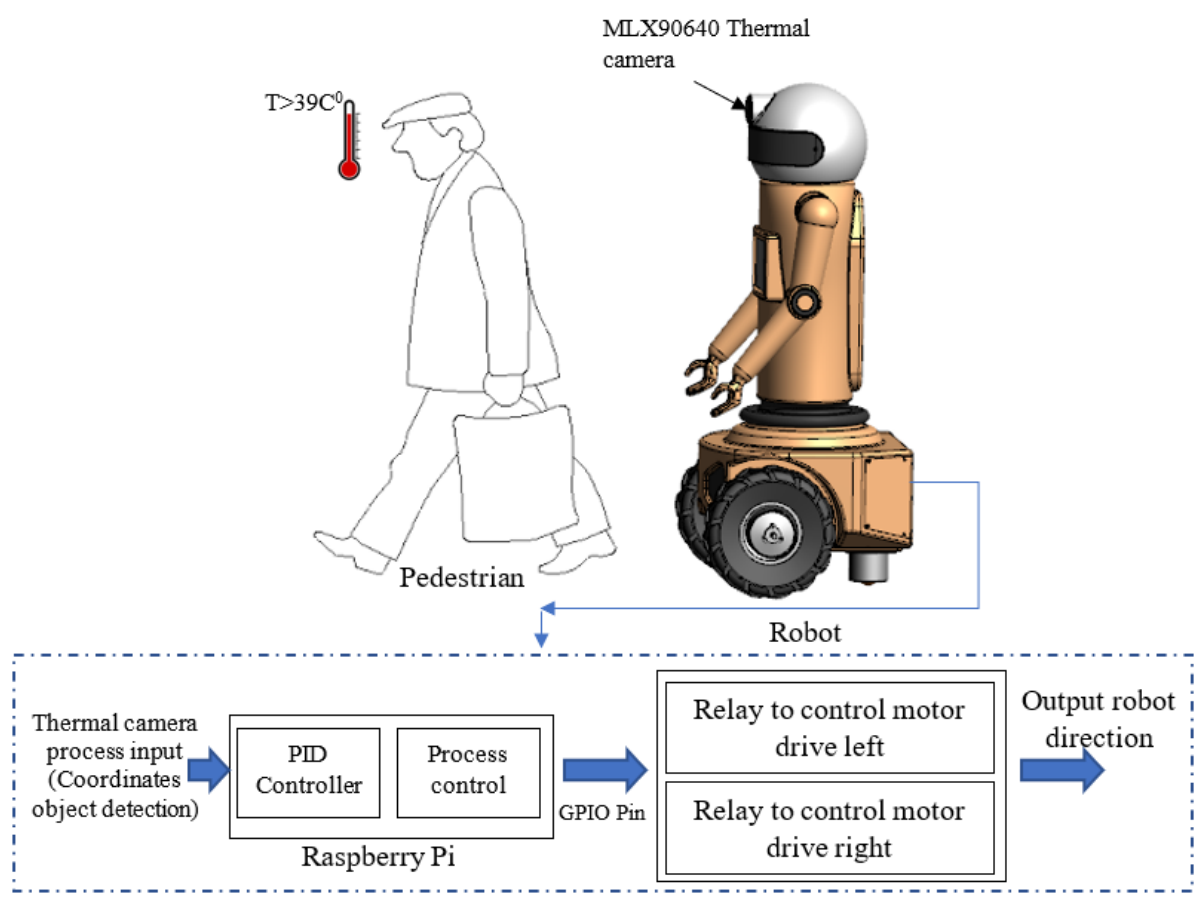

Fig. 3. CPR Robot tracking a target person has an abnormal temperature.

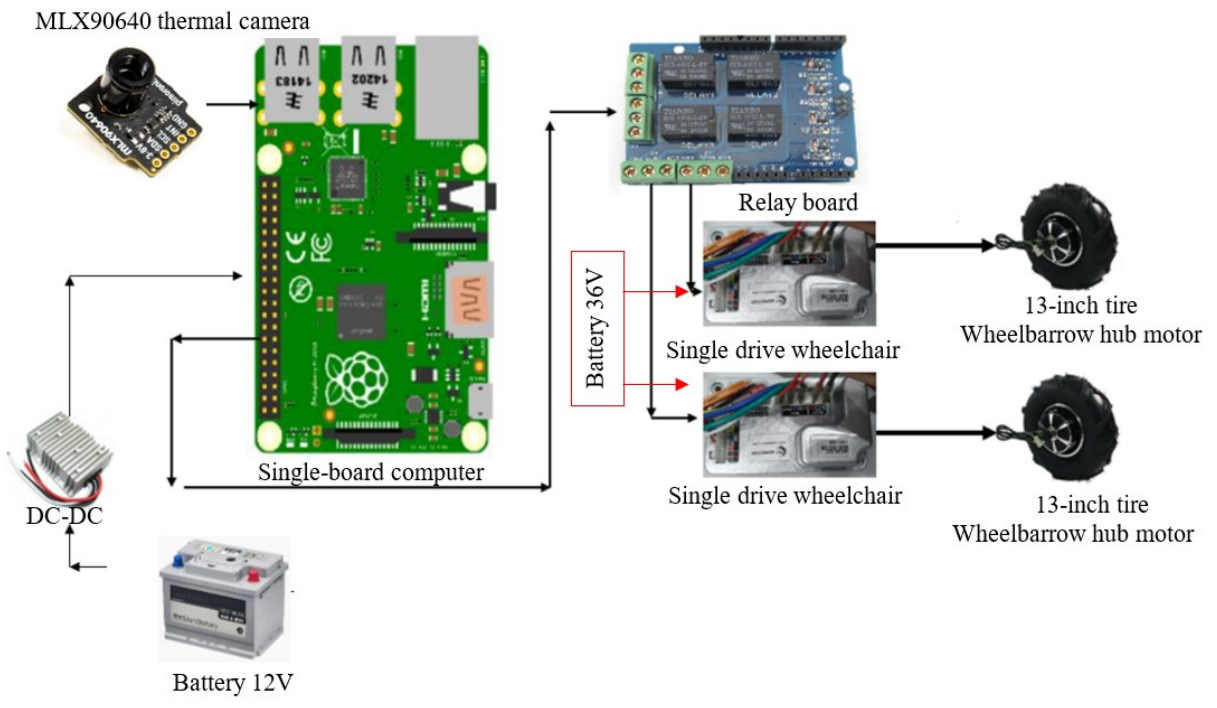

Fig. 4. The schematic diagram for the whole system 


\section{Human body temperature}

Since several countries have started implementing plans to re-open after vaccinating people and infection rates with the pandemic have decreased. Non-contact thermometers can be used as part of an initial screening at entry points to government buildings and public areas to identify and screen people who may have elevated temperatures. The thermal camera cannot detect a Covid-19. However, it is the best non-invasively can detect abnormal body temperature in a private and public area to alert security teams to check more with healthcare teams. People affected with Covid-19 have had a wide range of symptoms reported like fever and high body temperature [29]. We know that when we use thermometers correctly, temperature assessment may have an impact on limiting the spread of Covid-19 infection. Body temperature varies throughout the day. It is controlled in the center of thermoregulation in the anterior hypothalamus [30], [31]. Therefore, measuring body temperature can give information about the conditions of the human body. There are many types of fever, such as persistent and recurrent fever. A high fever is usually associated with a bacterial infection and lowered with a viral infection. There are various causes of fever, such as mononucleosis, virus, pneumonia, endocarditis, abdominal abscess, and meningitis. A particular type of fever occurs in the case of sepsis. In addition, some medications may cause fever. According to $\mathrm{WHO}$, any passenger has a body temperature higher than $39^{\circ} \mathrm{C}$; maybe he affected by Covid-19, so he should make Covid-19 test, as shown in Figure 5 .

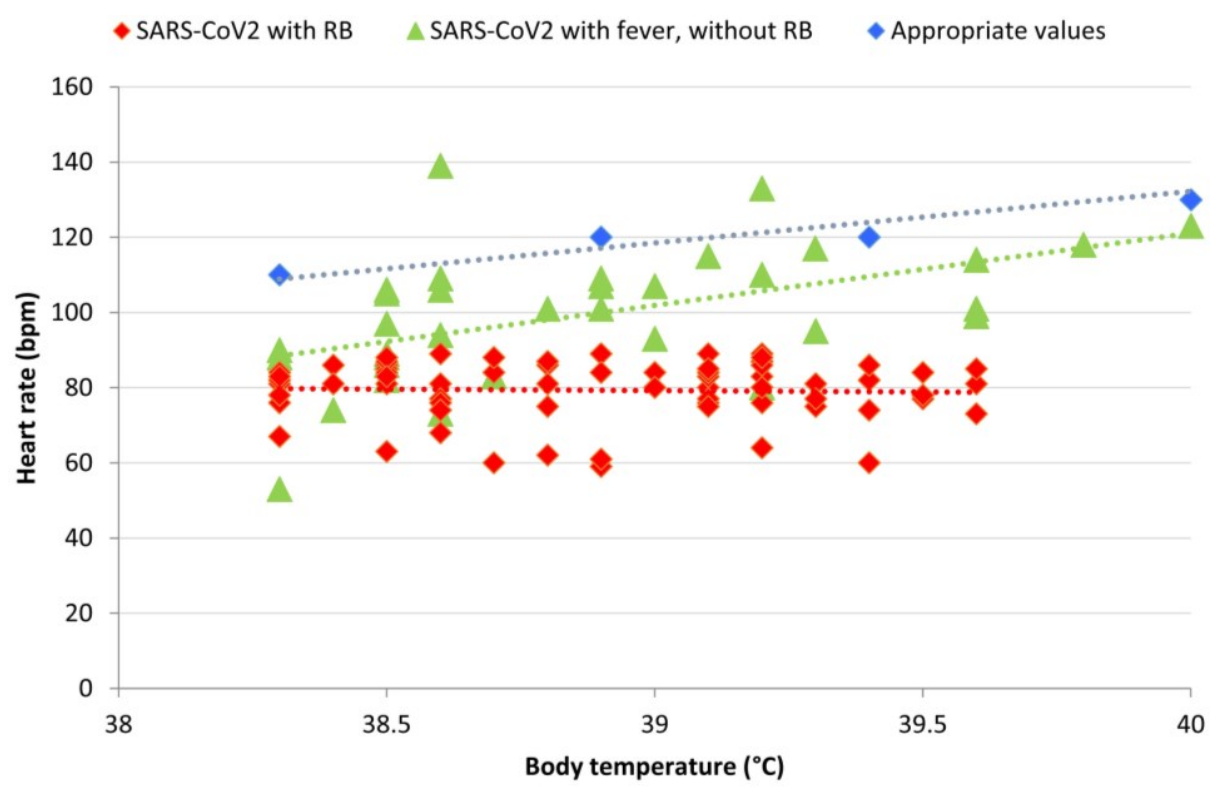

Fig. 5. Temperature-heart rate relationship in patients with Covid-19 [32] 


\subsection{Measurement methods}

Nowadays, most hospitals use independent non-contact body temperature measurements. It is an excellent method to measure the temperature for children, patients during sleep, and people affected by Covid-19 in the quarantine areas. Moreover, this technique provides a method measure of body temperature without contact with patients to reduce pandemic propagation.

\subsection{Skin temperature vs. internal body temperature}

There is a difference between human skin temperature and internal body temperature, known as basal temperature, and it varies across different parts of the body. For example, facial skin temperature is generally between $32^{\circ} \mathrm{C}$ (Cheek) and $34^{\circ} \mathrm{C}$ (Forehead), lower than the average normal body temperature due to heat exchange between the human body and the environment. To determine the internal temperature body via the skin temperature, we use the arterial heat balance method. Compensates for heat loss due to lower ambient temperature:

$$
T b=K(T s-T a)+T s
$$

Where:

- Tb: Body temperature,

- Ts: Skin temperature,

- Ta: Ambient temperature,

- $\mathrm{k}$ : Specific coefficient (Related to the skin measured, generally linked to blood perfusion in the tissue).

Studies have offered that the precision of the described technique is not ideal, but in our cases where precise temperature measurements are not required, like health checks to make further diagnostic.

\section{$3 \quad$ System design and implementation}

Recently, the number of researchers and projects on pedestrian tracking has been increasing. Much of this research uses a single board computer for digital signal processing, image processing, and robotic applications. In this research, a lightweight and compact thermal camera system can be used in the initial stage to diagnose and detect one of the signs of a pandemic, which is high body temperature. The MLX90640 thermal camera, 768 pixels ( $24 \times 32)$, can determine body temperature from about 2 meters. The MLX90640 thermal camera uses to obtain near-real-time temperature. This sensor converts the arithmetic value into a 17-bit ADC, which can be accessed using the I2C protocol to communicate with the computer unit. This camera measures the temperature of pedestrians in the government building with an accuracy of $0.02^{\circ} \mathrm{C}$. An image processing algorithm detects specific high temperatures in the Python environment and reduces noise in the rear of pedestrians. The image processing method aims to detect 
any object whose temperature exceeds more than $39^{\circ} \mathrm{C}$. This research determined the image processing method and implemented it in a robot model that uses artificial intelligence. Implementation of this robot very critical; very hard to detect the body temperature in a crowded area with high accuracy. The block diagram of the proposed robot, as is shown in Figure 6. The active power provides to a single-board computer and a thermal camera using a DC-DC converter.

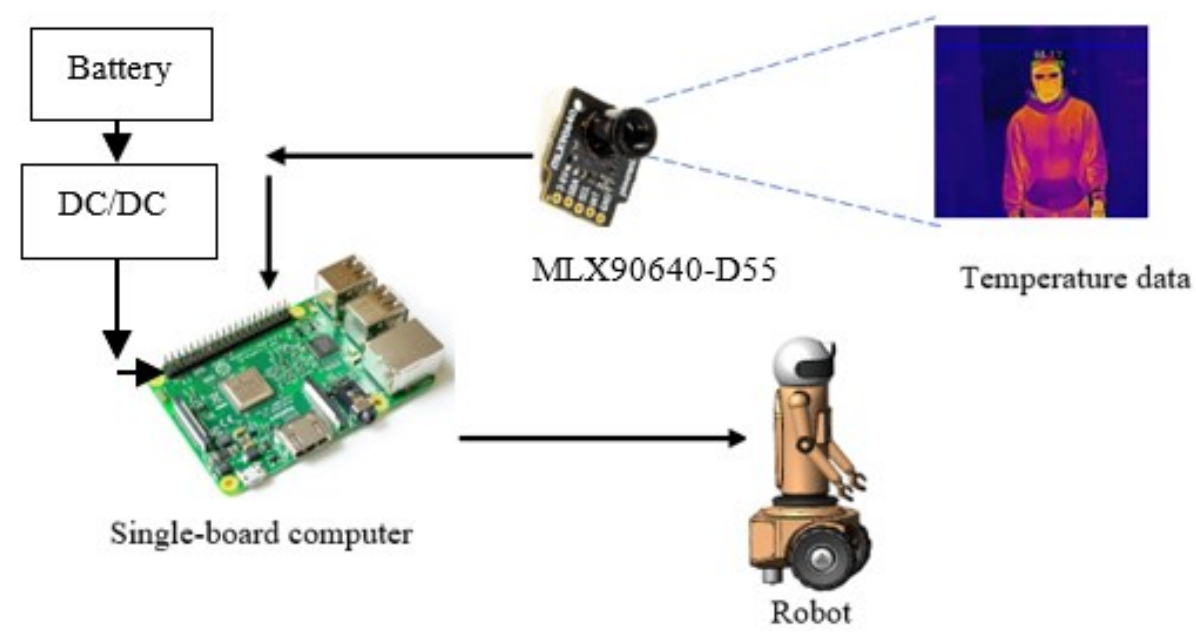

Fig. 6. Block diagram

The most significant difference is that MLX90640 cameras recognize and filter light beams such that only the infrared area of the electromagnetic spectrum is detected, not the visible zone. We design an algorithm to cope with this challenge. We choose this camera after making sure it is sensitive enough for slight temperature differences. To obtain reliable temperature information on pedestrians, it must be at least three times greater than the Field of View (FOV) of a single infrared detector used thermal imaging camera. The FOV of the single infrared detector depends on the distance between the lens and the pedestrian.

\subsection{MLX90640 thermal camera field of view}

There are two MLX90640 versions: One a wider FOV $\left(110^{\circ} \times 70^{\circ}\right.$ : MLX90640D110) and another one with a narrower FOV (55 $\times 35^{\circ}$ MLX90640-D55). The FOV is the angle confront by the sensor at the optical center of the lens. To determine the FOV value, we utilize the trigonometry equation (2), and all we need to do is use the procedure. It is vital to know that FOV follows the Focal Length of the Lens (FL) and relies on the spatial area of the camera sensor. The sensor senses from $-90^{\circ}$ to $90^{\circ}$ show a significant value at $0^{\circ}$, as shown in Figure 7. 

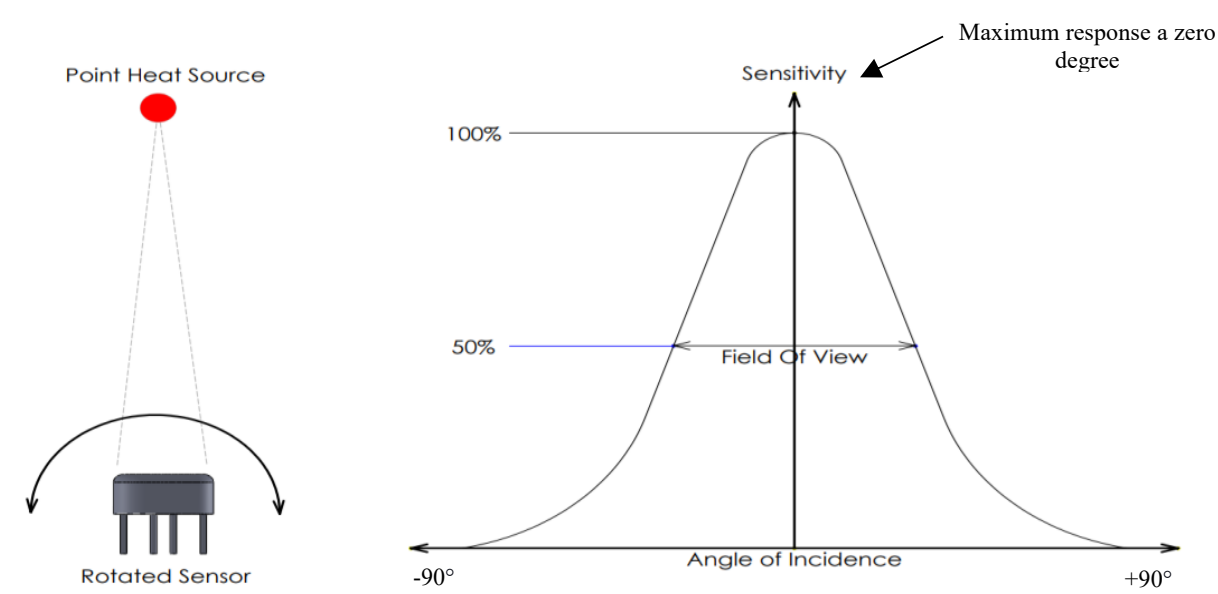

Fig. 7. Field of view (FOV) measurement

The Field of Views calculation using equation (2):

$$
F O V=2 \tan ^{-1} \frac{35}{2 * 50}=55^{\circ}
$$

The FOV must divide by the number of elements to get the HIFOV and VIFOV, using equations (3) and (4).

Where:

The HIFOV (Horizontal Instantaneous FOV)

$$
\text { HIFOV }=\frac{F O V}{32}=1.71^{\circ}
$$

The VIFOV (Vertical Instantaneous FOV)

$$
\text { VIFOV }=\frac{F O V}{24}=2.29^{\circ}
$$

The HIFOV and VIFOH values are roughly equal.

The body temperature defines by the MLX90640-D55 thermal camera, which has a viewing window on the red area, as shown in Figure 8 . The robot has a black spot area in the foreground equal to $460 \mathrm{~mm}$ and has an angle of $53.490^{\circ}$ horizontal FOV in this area where the body cannot be detected. The coordinates of the moving object seen by gaining the range of the detection area using the Pythagorean geometry method (5). The front view of the robot is approximately $2082 \mathrm{~mm}$ x $2000 \mathrm{~mm}$. This engineering calculation gives us the maximum that the robot can measure and track abnormal body temperature. For example, if the pedestrian is inside the black spot area, the robot will stop moving, and the same if the pedestrian is outside the FOV area.

$$
X=2000 * \tan \left(\frac{55}{2}\right)=1041 \mathrm{~mm}
$$




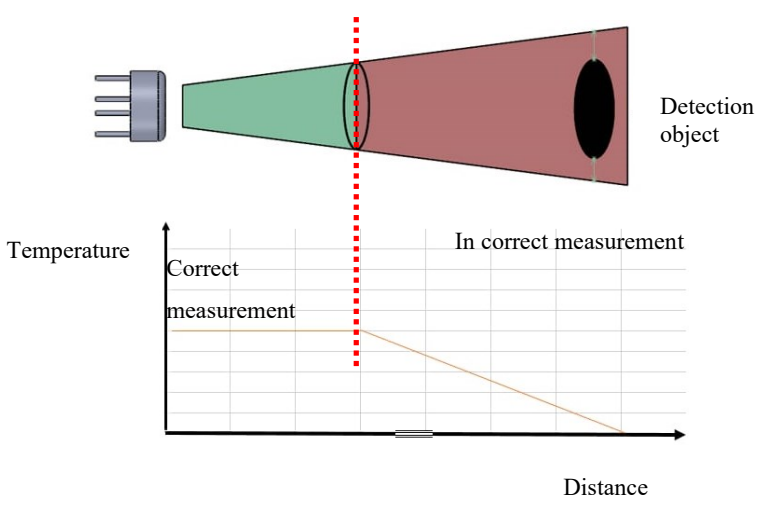

Fig. 8. Measured object distance

We determine the FOV by the angle in each sensor sensitive radiation, which means the sensor detects if the object in FOV. To determine the distance between sensors and objects, we use equation (6), as shown in Figure 9.

$$
D=\frac{S}{2 * \operatorname{Tan}\left(\frac{F O V}{2}\right)}
$$

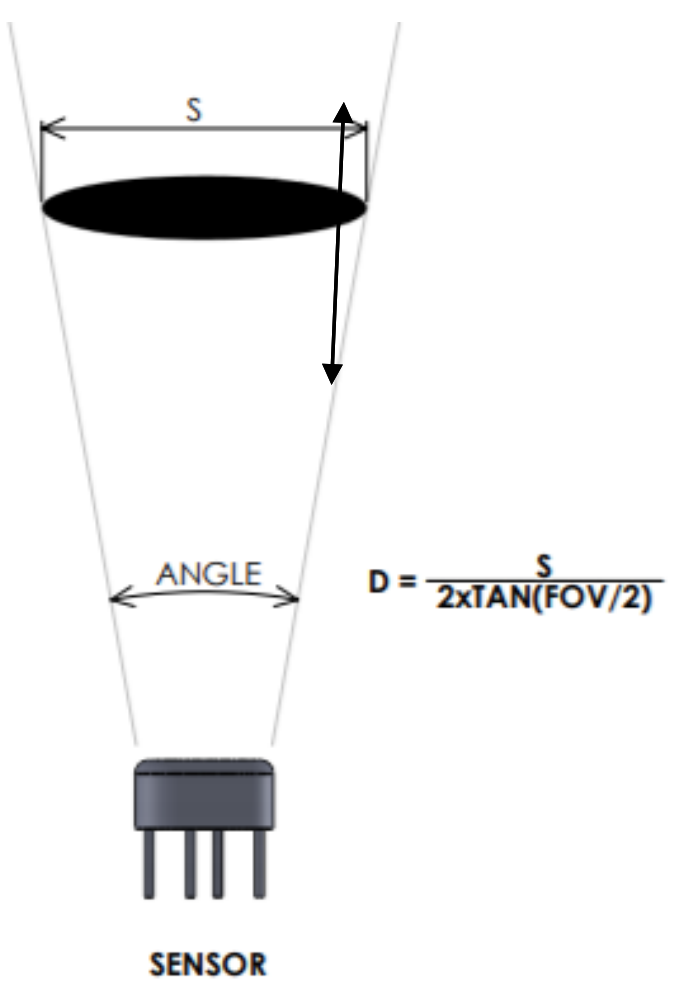

Fig. 9. MLX90640-D55 distance between sensors and objects 
This engineering estimate gives us the maximum amount that a robot can determine at a temperature level and at a level that tracks people. For example, if the object is within the empty spot area, the robot will stop moving, the same if the object is outside the observation field, as shown in Figure 10.

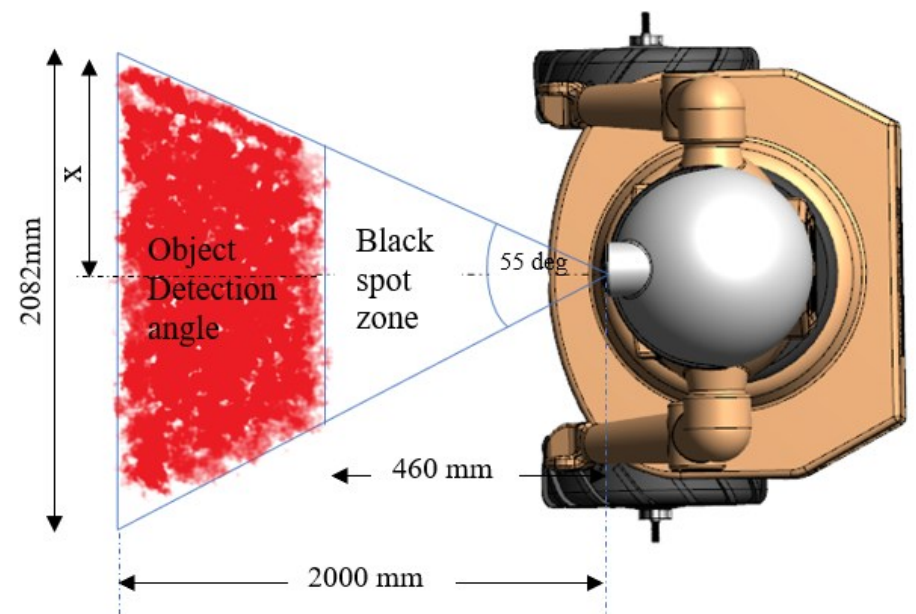

Fig. 10. Camera detection zone (Robot Top view)

The abnormal temperature was determined using a thermal camera. We utilize the I2C protocol to arrange the communication between the camera and the computer unit [33], [34], as shown in Figure 11.

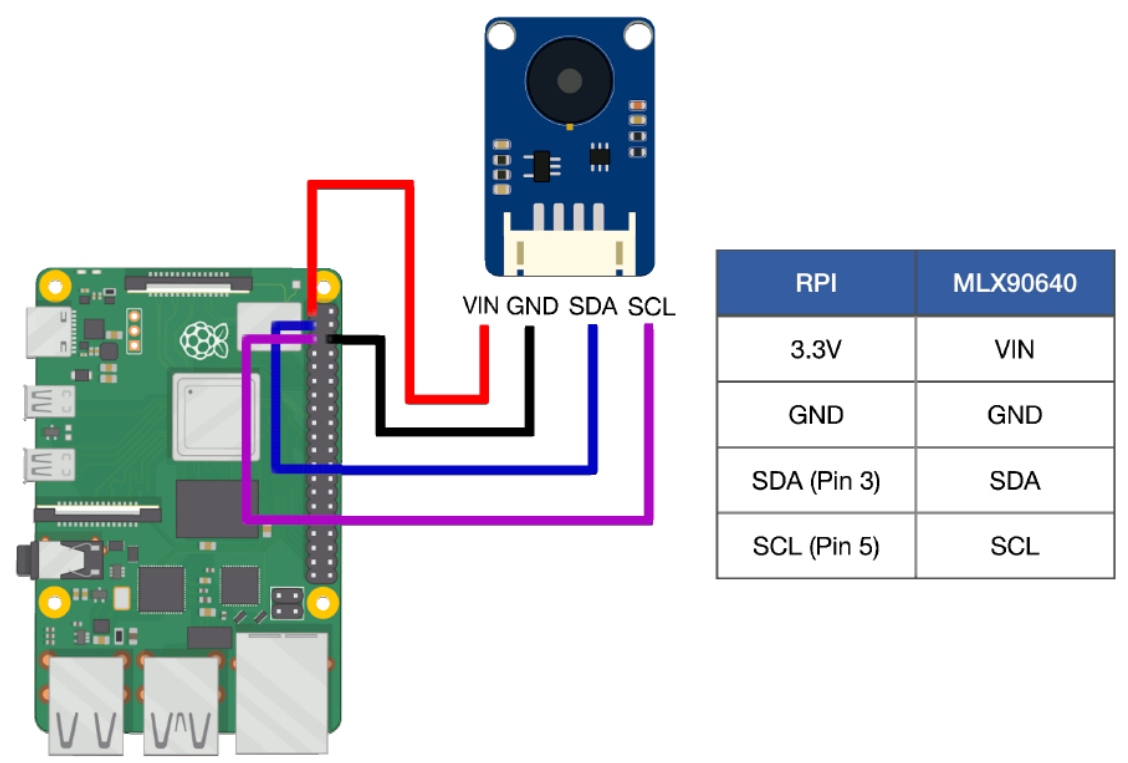

Fig. 11. Connecting the MLX90640 to a raspberry Pi3 


\subsection{Robot algorithm}

In this paper, a robot comprises several hardware components for testing and validation in the actual test. First, we monitor the temperature of pedestrians in public places for early diagnosis of abnormal body temperature. This section describes a strategy for identifying and tracking abnormal body temperature in thermal images. Finally, we implemented the algorithm, as shown in Figure 12.

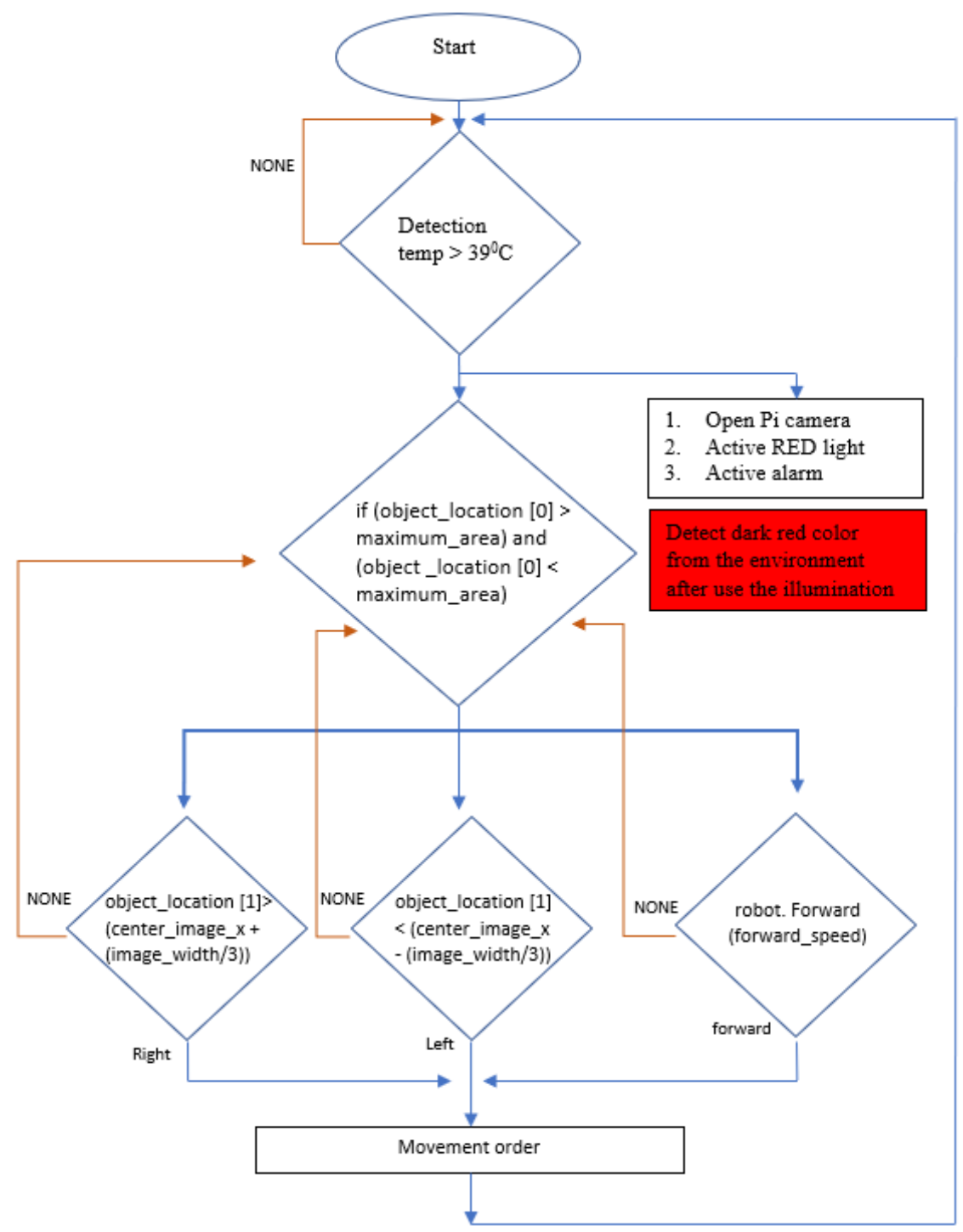

Fig. 12. Flow chart of the fever detection and tracking algorithm 
The objective of the experiments is to validate the feasibility of the proposed approach and the selected system architecture (including the thermal camera) for fever detection. The first part describes the fever detection task, which involves classifying the subject database into three categories. These three categories are:

- Low temperature (Hypothermia),

- Average temperature

- High temperature (Hyperpyrexia).

We compare the accuracy of the proposed system with the existing systems by measuring the temperatures of the forehead in a reasonable manner, which is the basic fact. Then, we calculate the standard deviation and variance of our reading. Finally, we calculate how close the distance reading is to the temperature reading from the forehead thermometer. Data for this experiment were acquired using frames containing images of multiple subjects' faces using an infrared camera. As for the second part, the robot will use data generated by an MLX90640 camera to track a person. Flowchart of the proposed approach for estimating facial temperature using telemetry. We test the robot in different environmental conditions and two different locations during different times of the day to test the system's efficiency.

We present the general step of this algorithm in the following steps:

a) Take a photo and measure the pedestrian body temperature.

b) The temperature scale is estimated for each pixel according to color gradient distribution.

c) If the temperature of the scanning bodies on the thermal image higher than $39^{\circ} \mathrm{C}$, Jump to 4 else Jump to 1 (average temperature)

d) A red led flashing with low-frequency synchronous with alarm is used to indicate the pedestrian has a fever.

e) If (pedestrian temperature $>$ minimum area) and (pedestrian temperature $<$ maximum area)

f) If body temperature detection $>$ (center image+(image-width/3)), the robot goes to right direction.

g) Else: pedestrian temperature $<($ center image $+($ image-width $/ 3))$, the robot goes to left direction.

h) Else: Body temperature detection in the center of the image, the robot goes to forward direction.

We measure the forehead temperature using a thermometer at the thermal video recording time to serve as our ground truth for comparison.

If all peoples are within normal temperature ranges, the robot stays on standby and measures the temperature of pedestrians; otherwise, the robot will follow a body that has an abnormal fever, and the system will sound alarmed for social distance increase the risk of cross-infection. A flashing red LED and an alarm to sign when a high fever condition a detected. 


\section{Robot prototype discussion}

The robot is equipped with two cameras to monitor pedestrians. In addition, the robot is equipped with ultrasonic sensors to detect obstacles and equipped with a voice broadcast system for guiding the peoples, as shown in Figure 13.

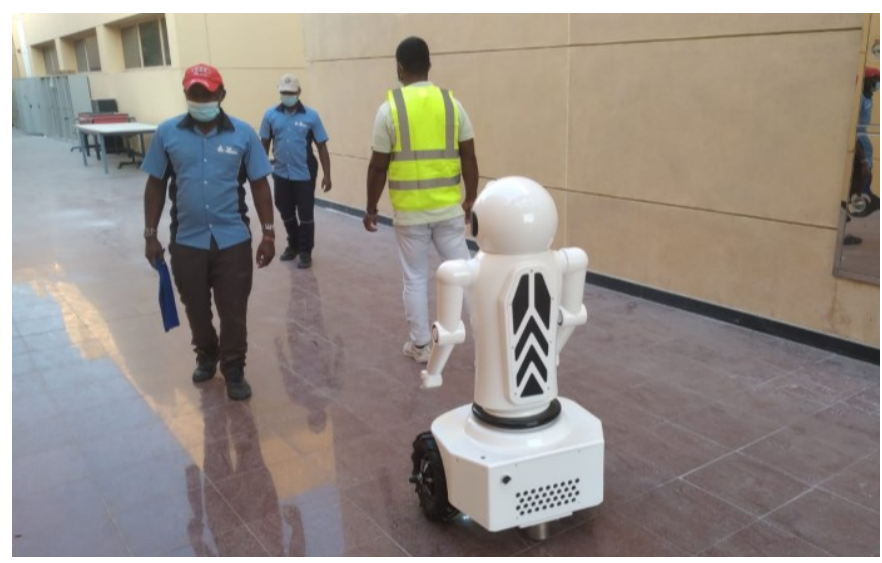

Fig. 13. Assembled and operational robot

The general system algorithm offerings the following flowchart. After the video analyses and transferred to the color space and provided the coordinate pedestrian position distance and angle value in part 1, the data was sent to part 2 to control the robot platform (Follow, Rotation right, or Rotation left), as shown in Figure 14.

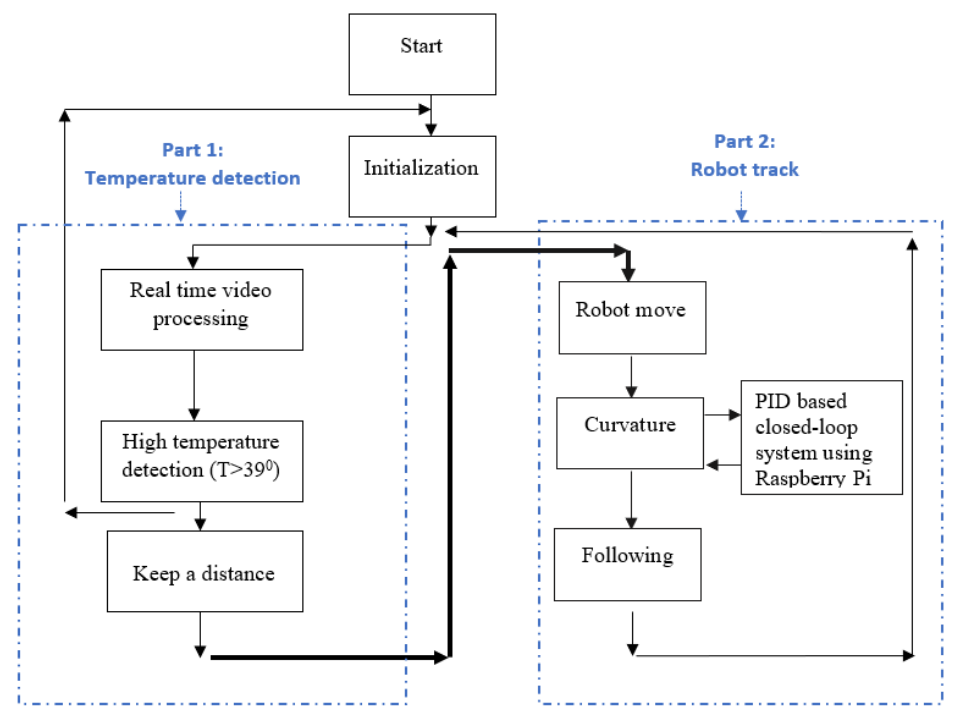

Fig. 14. Flow chart of the fever detection algorithm 
In Table 1, the robot hardware, and mechanical specifications.

Table 1. Hardware specifications of the robot

\begin{tabular}{|l|c|}
\hline List & Technical specification \\
\hline Robot size & $1460 \mathrm{~mm} \times 730 \mathrm{~mm} \times 450 \mathrm{~mm}$ \\
\hline Distance between tires $(\mathrm{mm})$ & 560 \\
\hline The radius of the tire $(\mathrm{mm})$ & 165.1 \\
\hline
\end{tabular}

\subsection{Moving robot}

This robot was designed in our lab and then printed and manufactured in China: the assembly and programming phase in our lab at the Qatar Science Club, as shown in Figure 15. 3D printing is the best and low-cost solution with short prototyping time throughput. In this paper, a three-dimensional two-wheeled robot is ready for testing. When the MLX90640 camera detects an abnormal human temperature, the robot will automatically follow the person concerned. It moves at the same speed with two degrees of freedom. We use the MLX90640 thermal camera to detect pedestrians and interact with the computer to do the action.
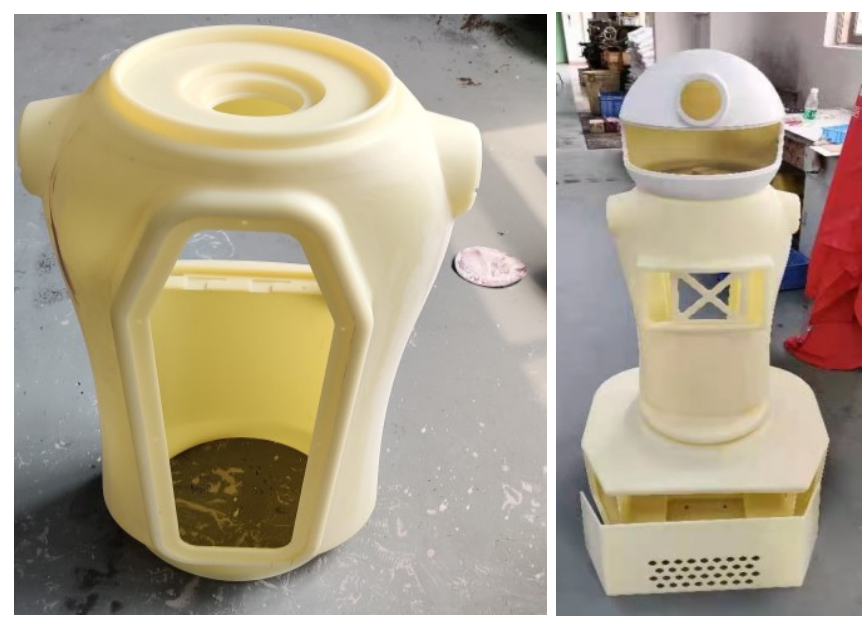

Fig. 15. 3D printed shell.

\subsection{Moving robot}

The robot mechanical structure contains many parts; the base consists of a two-wheel differential drive system (Hub motor 13") in the front, a flange-fitting ball in the backside, and batteries, as shown in Figure 16. We design the body to keep the camera sensor on the top of the robot is mounted on the robot head. The camera position is near a pedestrian height to better visual data with low noise. So initially, the camera location is set up at $1350 \mathrm{~mm}$. 


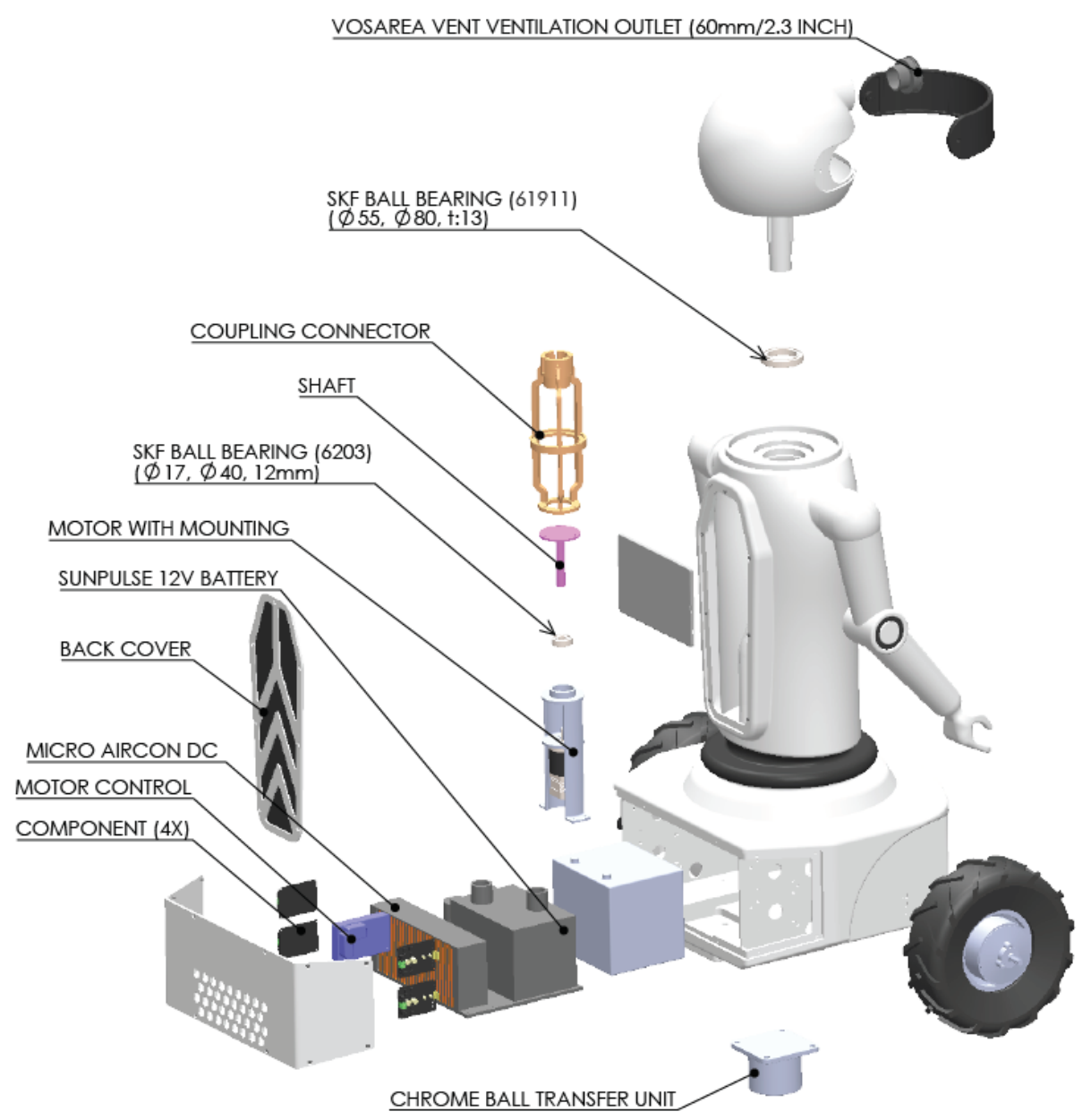

Fig. 16. Robot exploded components.

The system design comprises a computer unit and a control unit. The processing unit only uses a camera and is linked with the control unit to transmit the visual information after bulk processing serially. The control unit relates to the computer, and it makes uses an ultrasonic sensor. The ultrasonic sensor and camera work in unison with each other and helps the robot in its operation and navigate its path by avoiding obstacles and maintaining a specific distance from the object. The result is made based on information obtained from the sensor. The generalized system design is incorporating a camera and sensor. The processor that we have used is the Raspberry Pi single-board computer. After detecting high body temperature, the next phase establishes communication between the processor and control unit. We have used a relay board as a control unit. A relay-based motor driver module uses to control the motor drive. The advantage of this relay-based module is that it can quickly drive the high torque motors that require large fluxes. 


\section{$5 \quad$ Results and discussion}

The robot screening system has been used in our corridor, as shown in Figure 17. However, the developed screening system can also be applied to airports, metro stations, highways, schools, banks, supermarkets, and any other place requiring automatic follow abnormal body temperature. We can extract a thermal image from the MLX90640 camera, use the maximum surface temperature of the face region in this study, and the background temperature of the human, as shown in Figure 18. Photo (a) and (b) show an excellent performance of the camera. Pedestrian presence has been effectively detected, with an appropriate deviation of measured body temperature in two meters. A human has also been appropriately detected, with a clear difference between a human and background and good temperature measurement in the same distance, as shown in photos (c) and (d). According to the presented images (a), (b), (c), and (d), we concluded that the performance of sensor array MLX90640 is at a perfect level, appropriate for Covid-19 preventive detection.

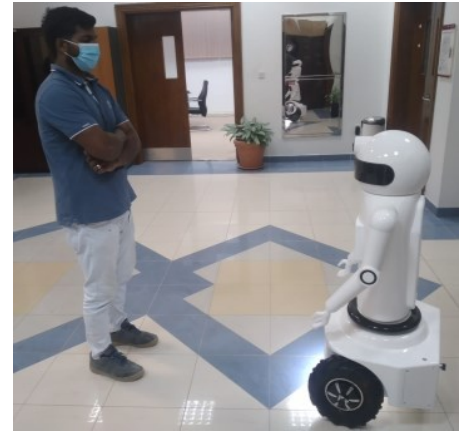

(a)

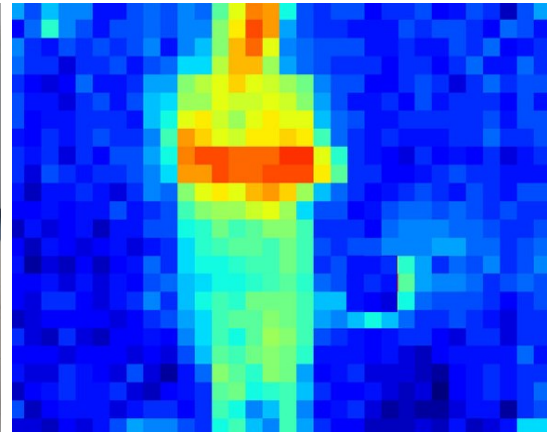

(b)

Fig. 17. (a) Reality human frond side; (b) Infrared array output of the sensor

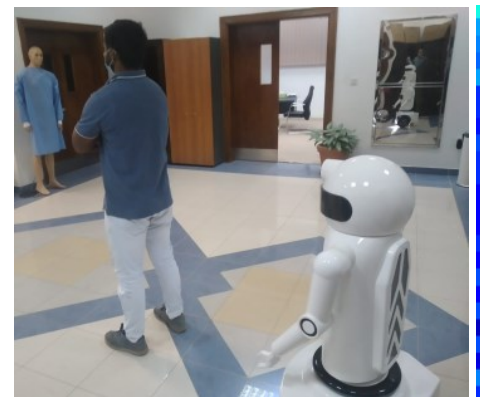

(c)

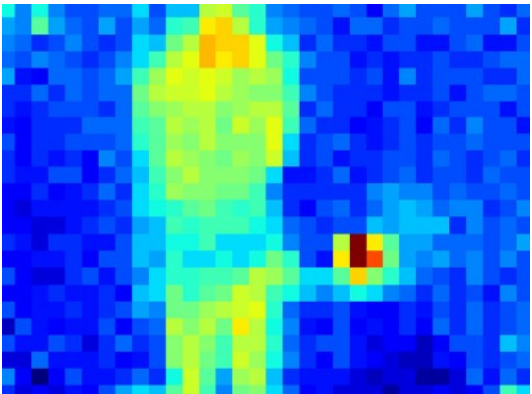

(d)

Fig. 18. (c) Reality human backside; (d) Infrared array output of the sensor 
To acquire the color filter process capabilities, we test the performance of color filtering. The tests focus on detecting dark red color from the environment after use the illumination filter. We carried a running experiment in the corridor of our department. In this test phase, the target person turned once right and left. The experiment phase gives us more idea about the ac curacy of the proposed algorithm and the efficiency of the robot prototype design. We often carried out the following experiment and measured the error distance and trajectory between the robot and the target person, as shown in Figure 19.

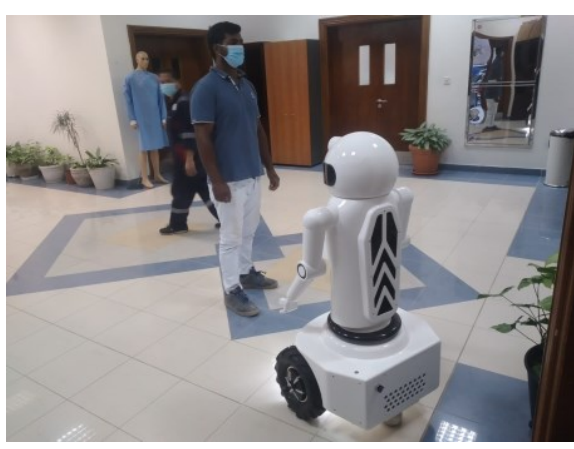

(a)

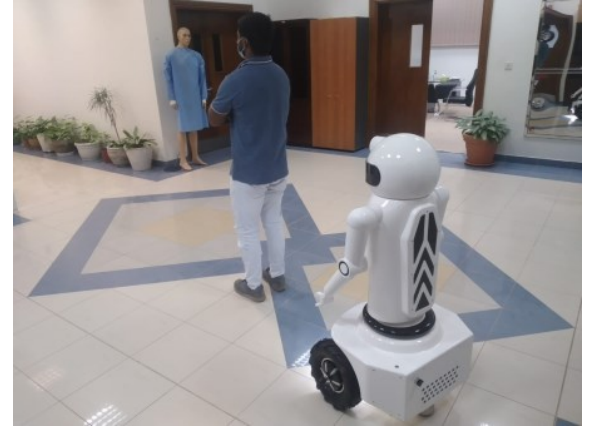

(b)

Fig. 19. Following a human in indoor areas. (a-b) different scenes.

In this photo, from (a) to (b), the robot follows the target smoothly, and the robot shows high flexibility to move and follow with low error. Unfortunately, the robot has not been tested on many people in different areas and workplaces; we will work on that in the future.

\section{Conclusion}

The purpose of this study is to use a high-tech technique to detect abnormal temperatures in a crowded area to reduce the spread of the coronavirus. We use this robot to measure the temperature of pedestrians, especially in open areas. This robot can follow a person using a thermal camera and robotic platform, and a precise tracking system. In this robot we used a low-cost infrared camera MLX90640 with a resolution of $\pm 2{ }^{\circ} \mathrm{C}$ and a resolution of $32 \times 24$ pixels. They were used to predict thermal sensation with a better performance of a skin temperature measurement system including thermocouples at different points of the skin with an accuracy of $\pm 0.15^{\circ} \mathrm{C}$.

Sensor's performance has been tested in the presence of humans and objects at temperatures close to ambient temperature. Most of the tests were conducted inside the QSC lane at different sensor distances ranging from $0.5 \mathrm{~m}$ to $2 \mathrm{~m}$. We have obtained thermal images of excellent quality in all cases by applying an algorithm that programmatically increases the resolution of the image and makes it smoother. The robot has an excellent ability to follow pedestrians in crowded areas, vibrant environments and 
indoors. To enable the robot to track a person with an abnormal body temperature in near real time, using image processing algorithms. We control the robot platform using a PID controller based on the detection of a person's coordinates through the camera installed in the robot's head.

The main idea is preventive to reduce the spread of Covid-19 in the social sphere. In addition, we can use this technology after further development and testing in other areas. Finally, we showed how such a sensor could remotely read body temperature using a single panel computer.

\section{$7 \quad$ References}

[1] AuthorsM. Şahin, "Impact of weather on COVID-19 pandemic in Turkey," Science of The Total Environment, vol. 728, p. 138810, Aug. 2020, https://doi.org/10.1016/j.scitotenv.2020.138810

[2] T. Alon, M. Doepke, J. Olmstead-Rumsey, and M. Tertilt, "The Impact of COVID-19 on Gender Equality," National Bureau of Economic Research, Working Paper 26947, Apr. 2020. https://doi.org/10.3386/w26947

[3] O. M. Al-Quteimat and A. M. Amer, "The Impact of the COVID-19 Pandemic on Cancer Patients," Am J Clin Oncol, Apr. 2020, https://doi.org/10.1097/COC.0000000000000712

[4] H. He and L. Harris, "The impact of Covid-19 pandemic on corporate social responsibility and marketing philosophy," Journal of Business Research, vol. 116, pp. 176-182, Aug. 2020, https://doi.org/10.1016/j.jbusres.2020.05.030

[5] C. A. Latkin, L. Dayton, G. Yi, B. Colon, and X. Kong, "Mask usage, social distancing, racial, and gender correlates of COVID-19 vaccine intentions among adults in the US," PLOS ONE, vol. 16, no. 2, p. e0246970, Feb. 2021, https://doi.org/10.1371/journal.pone. $\underline{0246970}$

[6] A. Moehring, A. Collis, K. Garimella, M. A. Rahimian, S. Aral, and D. Eckles, "Surfacing Norms to Increase Vaccine Acceptance," Social Science Research Network, Rochester, NY, SSRN Scholarly Paper ID 3782082, Feb. 2021. https://doi.org/10.2139/ssrn.3782082

[7] H. Ritchie et al., "Coronavirus Pandemic (COVID-19)," Our World in Data, Mar. 2020, Accessed: Aug. 06, 2021. [Online]. Available: https://ourworldindata.org/covid-vaccina$\underline{\text { tions }}$

[8] D. Laborde, C. Smaller, and J. Porciello, "What would it cost to avert the COVID-19 hunger crisis?," Jun. 2020, Accessed: Jun. 16, 2021. [Online]. Available: https://ecommons.cornell.edu/handle/1813/70172

[9] A. Sumner, E. Ortiz-Juarez, and C. Hoy, "Precarity and the pandemic: COVID-19 and poverty incidence, intensity, and severity in developing countries," WIDER Working Paper, Working Paper 2020/77, 2020. https://doi.org/10.35188/unu-wider/2020/834-4

[10] G. Valensisi, "COVID-19 and Global Poverty: Are LDCs Being Left Behind?," Eur J Dev Res, vol. 32, no. 5, pp. 1535-1557, Dec. 2020, https://doi.org/10.1057/s41287-020-00314-8

[11] E. Shim, "Optimal Allocation of the Limited COVID-19 Vaccine Supply in South Korea," Journal of Clinical Medicine, vol. 10, no. 4, Art. no. 4, Jan. 2021, https://doi.org/10.3390/ jem10040591

[12] K. M. Bubar et al., "Model-informed COVID-19 vaccine prioritization strategies by age and serostatus," Science, vol. 371, no. 6532, pp. 916-921, Feb. 2021, https://doi.org/10.1126/science.abe6959 
Paper-A Prototype of a Robot Capable of Tracking Anyone with a High Body Temperature in...

[13] C. Aschwanden, "Five reasons why COVID herd immunity is probably impossible," Nature, vol. 591, no. 7851, Art. no. 7851, Mar. 2021, https://doi.org/10.1038/d41586-021-00728-2

[14] "Covid-19: Israel records no daily deaths for the first time in 10 months," BBC News, Apr. 24, 2021. Accessed: Jun. 10, 2021. [Online]. Available: https://www.bbc.com/news/worldmiddle-east- 56868383

[15] "Total Covid-19 recoveries increase to 224,530: MoPH," Gulf-Times, Aug. 06, 2021. https://gulf-times.com/story/697952 (accessed Aug. 06, 2021).

[16] "1 in 6 adults in Qatar received at least first Covid-19 vaccine: MoPH," Doha News | Qatar, Mar. 22, 2021. https://www.dohanews.co/1-in-6-adults-in-qatar-received-at-least-firstcovid-19-vaccine-moph/ (accessed Aug. 06, 2021). https://doi.org/10.22541/au. $162041666.65803989 / \mathrm{v} 1$

[17] "Qatar: the latest coronavirus counts, charts and maps." https://graphics.reuters.com/worldcoronavirus-tracker-and-maps/countries-and-territories/qatar/ (accessed Jun. 16, 2021).

[18] "COVID19 Home." https://covid19.moph.gov.qa/EN/Pages/default.aspx (accessed Aug. 06, 2021).

[19] M. Z. Chaari, R. Al-Rahimi, M. Abdelfatah, and A. S. Khamis, "Use of reverse engineering method for respirator devices in COVID-19 crisis," in 2020 2nd International Conference on Electrical, Control and Instrumentation Engineering (ICECIE), Nov. 2020, pp. 1-4. https://doi.org/10.1109/icecie50279.2020.9309686

[20] M. Z. Chaari, M. Abdelfatah, C. Loreno, and R. Al-Rahimi, "Development of Air Conditioner Robot Prototype That Follows Humans in Outdoor Applications," Electronics, vol. 10, no. 14, Art. no. 14, Jan. 2021, https://doi.org/10.3390/electronics 10141700

[21] D. He et al., "Research on Temperature Calculation Method of Electrical Equipment Based on IR Data Compensation," IOP Conf. Ser.: Earth Environ. Sci., vol. 440, p. 032112, Mar. 2020, https://doi.org/10.1088/1755-1315/440/3/032112

[22] T. Bhowmik, R. Mojumder, I. Banerjee, G. Das, and A. Bhattacharya, "IoT Based NonContact Portable Thermal Scanner for COVID Patient Screening," in 2020 IEEE 17th India Council International Conference (INDICON), Dec. 2020, pp. 1-6. d https://doi.org/10. $\underline{1109 / \text { indicon } 49873.2020 .9342203}$

[23] P. Du, L. She, Y. Wang, S. Chang, and H. Li, "Design of intelligent air cooling radiator system based on TMS320C6748," in 2020 39th Chinese Control Conference (CCC), Jul. 2020, pp. 2821-2826. https://doi.org/10.23919/ccc50068.2020.9188721

[24] V. Tsvetkov, G. Spasov, and G. Petrova, "IoT solution for monitoring of data in the visible and infrared spectrum," in 2020 XXIX International Scientific Conference Electronics (ET), Sep. 2020, pp. 1-4. https://doi.org/10.1109/et50336.2020.9238289

[25] K. Kapás, T. Bozóki, G. Dálya, J. Takátsy, L. Mészáros, and A. Pál, “Attitude determination for nano-satellites - I. Spherical projections for large field of view infrasensors," Exp Astron, Apr. 2021, https://doi.org/10.1007/s10686-021-09730-y

[26] N. S. Yamanoor, S. Yamanoor, and K. Srivastava, "Low Cost Design of Non-Contact Thermometry for Diagnosis and Monitoring," in 2020 IEEE Global Humanitarian Technology Conference (GHTC), Oct. 2020, pp. 1-6. https://doi.org/10.1109/ghtc46280.2020.9342853

[27] Z. Chen and Y. Wang, "Remote Recognition of In-Bed Postures Using a Thermopile Array Sensor With Machine Learning," IEEE Sensors Journal, vol. 21, no. 9, pp. 10428-10436, May 2021, https://doi.org/10.1109/jsen.2021.3059681

[28] K. Dimitrov, V. Shterev, and T. Valkovski, "Low-cost system for recognizing people through infrared arrays in smart home systems," in 2020 XXIX International Scientific Conference Electronics (ET), Sep. 2020, pp. 1-4. https://doi.org/10.1109/et50336.2020. $\underline{9238246}$ 
Paper-A Prototype of a Robot Capable of Tracking Anyone with a High Body Temperature in...

[29] L. Li et al., "Characteristics and serological patterns of COVID-19 convalescent plasma donors: optimal donors and timing of donation," Transfusion, vol. 60, no. 8, pp. 1765-1772, 2020, https://doi.org/10.1111/trf.15918

[30] E. Bozkurt, "RECENT ADVANCES IN ACUTE PANCREATITIS," 2021, pp. 131-138.

[31] J. Kang et al., "Predicting the location of the preoptic and anterior hypothalamic region by visualizing the thermoregulatory center on fMRI in craniopharyngioma using cold and warm stimuli," Aging (Albany NY), vol. 13, no. 7, pp. 10087-10098, Mar. 2021, https://doi.org/ 10.18632/aging.202766

[32] G. Capoferri, M. Osthoff, A. Egli, M. Stoeckle, and S. Bassetti, "Relative bradycardia in patients with COVID-19," Clinical Microbiology and Infection, vol. 27, no. 2, pp. 295-296, Feb. 2021, https://doi.org/10.1016/j.cmi.2020.08.013

[33] V. Chidurala and X. Li, "Occupancy Estimation Using Thermal Imaging Sensors and Machine Learning Algorithms," IEEE Sensors Journal, vol. 21, no. 6, pp. 8627-8638, Mar. 2021, https://doi.org/10.1109/jsen.2021.3049311

[34] V. Manuel Ionescu and F. Magda Enescu, "Low cost thermal sensor array for wide area monitoring," in 2020 12th International Conference on Electronics, Computers and Artificial Intelligence (ECAI), Jun. 2020, pp. 1-4. https://doi.org/10.1109/ecai50035. $\underline{2020.9223193}$

\section{Authors}

Dr. Mohamed Zied Chaari, Qatar Scientific Club, Doha, Qatar (Department: Fabrication laboratory).

Eng. Abdulaziz Aljaberi, Qatar Scientific Club, Doha, Qatar (Department: Fabrication laboratory).

Article submitted 2021-07-12. Resubmitted 2021-08-06. Final acceptance 2021-08-06. Final version published as submitted by the authors. 